TRANS · núm. I4 · 2010

ARTICULOS · 63-82
Este artículo aborda el estudio de la traducción literal como estrategia de desterritorialización de la lengua y caso especial de alternancia de lenguas en la colección de relatos Woman Hollering Creek de la autora chicana Sandra Cisneros. Con el propósito de explorar esta estrategia de desterritorialización, examinaremos una selección de expresiones idiomáticas que, situadas en los niveles léxico-semántico y pragmático del discurso, se perciben como traducciones literales del español al inglés. Dada la relación que existe entre la desterritorialización de la lengua, la alternancia de lenguas y la traducción literal en la construcción del discurso de Cisneros, presentaremos dos modelos sociolingüísticos complementarios (Myers-Scotton, 1993a, 1993b), que pueden combinarse con la teoría de las heterogeneidades enunciativas (Authier, 1984, 1995) para dar cuenta de los casos de traducción literal presentes en esta narrativa.

PALABRAS CLAVE: desterritorialización, Sandra Cisneros, literatura chicana, traducción literal, alternancia de lenguas, heterogeneidades enunciativas

\title{
La traducción literal como estrategia de desterritorialización y caso especial de alternancia de lenguas en Woman Hollering Creek de Sandra Cisneros*
}

Literal Translation as a Strategy of Deterritorialization and Special Case of Code-switching in Sandra Cisneros's Woman Hollering Creek

This article approaches the study of literal translation as a strategy of deterritorialization and as a special case of code-switching in Woman Hollering Creek, a collection of stories by the Chicana writer Sandra Cisneros. In order to explore this strategy of deterritorialization, we will examine a selection of idiomatic expressions which, placed at the lexicalsemantic and pragmatic levels of discourse, are perceived as literal translations from Spanish to English. Given the relationship between the deter-

Maria Laura Spoturno

Universidad Nacional de La Platal ritorialization of language, code-switching and literal translation in the construction of Cisneros's discourse, we will present two complementary sociolinguistic models (Myers-Scotton, 1993a, 1993b) which might combine with the theory of enunciative heterogeneities (Authier, 1984, 1995) to account for the cases of literal translation found in this narrative.

KEY WORDS: deterritorialization, Sandra Cisneros, Chicano literature, literal translation, code-switching, enunciative heterogeneities 


\section{INTRODUCCIÓN}

En este trabajo, nos proponemos indagar en el 64 estatuto y la función de la traducción literal en la colección de relatos Woman Hollering Creek (199I) ${ }^{\mathrm{I}}$ de la autora chicana Sandra Cisneros (1954). Según nuestra hipótesis, en el seno de la narrativa de Cisneros, la traducción literal se presenta como una fuerte estrategia de desterritorialización de la lengua y constituye un caso especial de alternancia de lenguas. Antes de abordar el estudio de la traducción literal en el sentido propuesto, es necesario realizar ciertas precisiones que guiarán todo el desarrollo de este artículo y nos permitirán situar el ámbito de su incumbencia. En primer lugar, debemos delimitar el alcance del concepto de traducción literal y de desterritorialización. Siguiendo a Newmark (1988 [1982]), la traducción literal puede definirse como el proceso por medio del cual se vierten en una lengua y texto meta los sentidos primarios de las unidades léxicas de un texto fuente, fuera de todo contexto, respetando, no obstante, las estructuras sintácticas de esa lengua. Ahora bien, no nos ocuparemos aquí de la traducción literal en este sentido; es decir, este artículo no examina la operación traductológica que busca reproducir palabra por palabra los elementos léxicos de una obra literaria en una lengua meta determinada. Nuestro interés se centra en el empleo de la traducción literal como estrategia discursiva interna a un texto

* Este artículo forma parte de nuestro proyecto de investigación doctoral Heterogeneidad(es) enunciativa(s) y discursos argumentativos en la narrativa chicana: el caso de Sandra Cisneros, que desarrollamos en el marco de una Beca de Postgrado Interna del Consejo Nacional de Investigaciones Científicas y Técnicas (CONICET).

I Cisneros, S. (199I). Woman Hollering Creek and Other Stories. Nueva York: Vintage Contemporaries. Las referencias a esta colección de relatos se harán por medio de la abreviación $W H C$ y siguiendo la paginación de esta edición, que se indicará entre paréntesis dentro del mismo texto. literario, en el que esta se usa para generar efectos estéticos particulares y evocar sentidos lingüístico-culturales nuevos, sin que medie la intervención de dos textos. Ciertamente, en la narrativa de Cisneros, la traducción literal contribuye a la constitución de un discurso sumamente fronterizo ${ }^{2}$ y se vuelve un medio eficaz para «quitarle territorio» al inglés, la lengua mayoritaria en la que la autora escribe su obra literaria.

Por su parte, al definir la literatura de minorías, Deleuze y Guattari (1975) señalan la desterritorialización de la lengua como uno de sus tres rasgos distintivos. En íntima relación con la operación de desterritorialización, se sitúan las otras dos características esenciales de estas escrituras: el dispositivo colectivo de enunciación y el componente político. Como ha subrayado Martín-Rodríguez (1996), en el seno de las literaturas de minorías, la lengua minoritaria socava la lengua mayoritaria desde dentro del mismo sistema de la lengua, acción que tiene dos consecuencias principales: la desterritorialización de la lengua mayoritaria y la desintegración de la supuesta homogeneidad del sistema. De esta forma, se pone de manifiesto el fuerte componente político-ideológico que acompaña el trabajo de la escritura en el caso de estos autores. Aquí exploraremos un aspecto de la operación de desterritorialización en $W H C$ de la autora chicana, que se vincula justamente con el uso de la traducción literal.

Resulta de interés considerar, en segundo término, que la producción literaria de los autores del mundo poscolonial, al igual que la de los escritores de minorías como Cisneros, se juega en el espacio de lo que la teoría poscolonial ha denominado traducción cultural (Bhabha, 2004

2 En Spoturno (2008) nos ocupamos de la constitución del discurso fronterizo en $W H C$ de Sandra Cisneros. 
[1994])3. Este concepto, como bien apunta Trivedi (2005), no debe entenderse como una operación en la que intervienen dos textos, un texto fuente y un texto meta, ni como la traducción de elementos culturales. El término traducción cultural alude a la condición migrante del hombre y la mujer que deben ahora «traducirse» para ser en la realidad que les imponen el Tercer Mundo, los vestigios de las colonias y las inmigraciones; es decir, el contexto poscolonial actual. Así, y según propone Tymoczko (2000), resulta más pertinente pensar que los escritores del mundo poscolonial no escriben sino que «traducen» sus obras literarias recurriendo a originales que no existen sensu stricto.

En tercer término, y ya situados dentro del campo de los estudios chicanos, Rudin (I996) ha sugerido que el escritor chicano se presenta como un mediador cultural y traductor de diferencias culturales. Evocando las ideas de Scheliermacher (I8I3), Rudin plantea que el autor chicano puede, al igual que el traductor, adoptar dos posturas principales frente a la escritura: o bien acerca el lector al texto original y allí el efecto es de extranjerización, o bien decide acercar el texto al lector y genera así un efecto de domesticación. Rudin señala que en el caso de los escritores chicanos no existe un texto original, sino un texto cultural que el escritor traduce en su obra. Asimismo, Irizarry (200I) indica que estos textos se construyen en la alusión permanente a una

3 Resulta ineludible recurrir aquí a algunos de los postulados de la teoría poscolonial por cuanto su objeto de estudio guarda similitudes con las características propias de la literatura chicana, razón por la cual los críticos de la frontera, que investigan las obras chicanas, han hecho uso de nociones como hibridación, traducción cultural, entre otras. Las cuestiones teóricas que impone esta situación quedan fuera del alcance de este artículo. Para ampliar este tema, podrá consultarse: Aschcroft et.al (2002 [I989]), de Zavalía (2000), Scott and Michaelson (2003), entre otros. cosmovisión otra, que se asocia a lo latino 4 y asevera que bien podemos considerar estas obras como traducciones de originales fantasmagóricos.

Así, podemos afirmar que la traducción juega un rol preponderante en la construcción discursiva de la literatura chicana, en la que, como nos recuerda Arteaga (1997), siempre se evidencia la existencia de voces que compiten por el sentido $y$ constituyen fibras esenciales del entramado textual. En el caso particular de la narrativa de Cisneros, debemos apreciar que la autora chicana explota por medio de la traducción literal la potencialidad expresiva que se deriva de la puesta en escena de dos sistemas lingüísticoculturales diferentes, uno asociado a la cosmovisión latina y el otro, a la sajona, que fusiona con maestría en su prosa singular. El empleo creativo de las fisuras que se vislumbran en el encuentro de estos sistemas constituye, para Arteaga (op.cit.), un espacio privilegiado para la expresión de nuevos sentidos estéticos y culturales. Como veremos en las secciones siguientes, la traducción literal se vuelve un medio ideal para explorar nuevos terrenos discursivos.

Por último, es preciso tener presente que a lo largo de la historia de la literatura chicana las investigaciones han puesto el acento en dos estrategias de desterritorialización fundamentalmente: el uso del inglés para expresar conceptos vinculados a las comunidades chicana y mexicana y el recurso a la alternancia de lenguas para expresar ciertas cuestiones culturales (Lipski, 1982; Rudin, I996; Cintrón \& Mendieta-Lombardo, I995; Brady, I999; SolerEspiauba, 200I; Cantero \& Stewart, 2002;

4 En este artículo, emplearemos la palabra latino/a, en lugar del término hispano, en consonancia con la elección generalizada de las comunidades latinas de los EE. UU., que prefieren su uso, ya que este pone de relieve un vínculo entre sus comunidades y el resto de Latinoamérica. 
Jiménez Carrá, 2005; Leal y Martín-Rodríguez, 2006; García Vizcaíno, 2008; entre otros). Aquí, como ya hemos mencionado, nos interesa poner el énfasis en el estudio de la traducción literal como estrategia de desterritorialización y caso especial de alternancia de lenguas. Tal como mostraremos a continuación, este uso aparece esbozado en Rudin (1996) pero no explorado suficientemente.

Para intentar dar cuenta de este empleo singular de la traducción literal en $W H C$, presentaremos el análisis de un corpus constituido por frases y estructuras que registran cierta fijación. El interés por una selección de formas fijas reside en que, si bien se hallan expresadas en inglés, estas formas remiten al español como marco discursivo y se perciben como traducciones literales del inglés al español. Hemos realizado la selección y clasificación del corpus a partir de una hipótesis central que indica que bajo la apariencia de una única lengua, el inglés (LI), hay otra lengua, el español (L2), que se manifiesta en la utilización de alguno de sus niveles (léxicosemántico, pragmático, morfosintáctico o gráfico), lo cual contribuye a la desterritorialización de LI. Finalmente, el análisis de distintos casos ha puesto a prueba esta hipótesis que se sustenta en la noción de que estos cambios en los niveles, producidos a partir de la traducción literal, constituyen instancias especiales de alternancia de lenguas 5 .

La desterritorialización deja huellas, marcas, que resulta pertinente estudiar para explicar el carácter fronterizo de la obra de Cisneros. Precisamente, el empleo de formas que se perciben como traducciones literales del español al inglés constituye un punto fuerte de heterogeneidad y

5 Emplearemos el término «alternancia de lenguas» en lugar de «cambio de código» porque consideramos que la noción de lengua evoca el acervo cultural que está por detrás del código.

un medio para la desterritorialización de la lengua. Por otro lado, en el seno de esta narrativa, la marca de la traducción literal permite vislumbrar ese original imaginario asociado al mundo latino al que recurre la escritora a lo largo de su prosa. En ese solapamiento de imágenes y sistemas lingüístico-culturales se entrevé la presencia del español como elemento esencial de la trama discursiva.

Dada la relación que existe entre la estrategia de desterritorialización, la traducción literal y la alternancia de lenguas, examinaremos algunas nociones claves de dos modelos sociolingüísticos que abordan, de forma complementaria, la alternancia de lenguas en el habla de comunidades bilingües y multilingües (Myers-Scotton, I993a, I993b) con el propósito de evaluar la pertinencia de sus categorías al estudio que pretendemos realizar. Ahora bien, aun si estas investigaciones aportan conceptos esenciales para nuestro estudio, no contemplan dentro de sus descripciones los casos particulares relacionados con la traducción literal, que constituyen el objeto específico de nuestro trabajo.

Sostendremos así que la teoría de las heterogeneidades enunciativas (Authier-Revuz, 1984, 1995) propone los elementos necesarios para abordar el estudio de la traducción literal como estrategia de desterritorialización en la narrativa de Cisneros en tanto explica los procesos que se ponen en marcha en la constitución y representación del discurso y atiende especialmente a las marcas del discurso. En efecto, desde esta perspectiva teórica la traducción literal señala un punto de heterogeneidad, una alteridad a la que recurre el discurso en el proceso de su constitución.

En suma, en $\S$ I examinaremos sucintamente la desterritorialización de la lengua en $W H C$ de Cisneros para evaluar en $\S 2$ la posibilidad de aplicar dos modelos sociolin- 
güísticos al presente estudio. A continuación, presentaremos la perspectiva teórica que enmarca el análisis de casos: la teoría de las heterogeneidades enunciativas. En $\S 3$, con el objetivo de poner a prueba la hipótesis enunciada más arriba, ejemplificaremos el nivel morfosintáctico para luego analizar algunos aspectos de los niveles léxico-semántico y pragmático. Finalmente, en las Conclusiones expondremos las contribuciones y los posibles temas de investigación que surgen como consecuencia de este trabajo.

\section{ACERCA DE LA DESTERRITORIALIZA- CIÓN DE LA LENGUA EN LA NARRATIVA CHICANA}

Siguiendo a Deleuze y Guattari (I975), la literatura de minorías se define en oposición a la literatura canónica. Debe advertirse que no se trata de obras literarias escritas en un idioma minoritario, sino de una literatura que surge en el seno de una lengua y cultura mayoritarias o dominantes. Este es el fenómeno que se conoce como desterritorialización de la lengua: la lengua mayoritaria «pierde territorio» al ser empleada por los escritores de minorías. En estas literaturas se desdibuja la figura del sujeto; todo cobra un valor colectivo. La historia narrada representa las voces de todos, de una causa común. En términos generales, en la literatura canónica, el medio social es un trasfondo en el que las distintas acciones individuales se desarollan y relacionan. En el caso de la literatura de minorías, el problema individual se pone en la lente del microscopio y se vuelve inseparable del medio, vinculándose así ineludiblemente con el aspecto político.

Dentro de la producción literaria de las 1lamadas «minorías latinas», el estudio de obras chicanas se plantea como un desafío particu- lar porque inevitablemente nos remite a los distintos momentos que marcaron al pueblo chicano a lo largo de su historia. Es importante observar que la mayoría de estos momentos son acompañados de procesos relativos al contacto de lenguas. No será de asombro advertir que, en términos generales, la narrativa chicana constituye un discurso mestizo, de frontera, que pone de relieve la cuestión de la identidad lingüístico-cultural como una trama compleja en la que confluyen factores sociales, políticos, geográficos y lingüísticos.

Es posible afirmar que la narrativa chicana presenta una operación de desterritorialización doble. La primera fase de la desterritorialización se sitúa en el marco de la conquista de América en el siglo XVI principalmente. La segunda fase, tan compleja como la primera, se ubica en el marco de los conflictos y las contiendas por el territorio mexicano que hoy es parte del sudoeste de Estados Unidos ${ }^{6}$. El primer momento obligó al mundo mexicano antiguo a hablar la lengua del colonizador español y el segundo, a hablar otra lengua mayoritaria, el inglés. Es preciso notar que los mecanismos utilizados para imponer una lengua en una comunidad traen aparejadas situaciones lingüísticas relativas a la diglosia ${ }^{7}$ y a los fenómenos de

6 La realidad chicana ha sido explicada a partir de distintos modelos, entre los cuales es crucial considerar el modelo asimilacionista y el modelo del colonialismo interno. Para una presentación de estos modelos en relación con la literatura chicana, ver Staples (I976), Muñoz Jr. (I983) y Barrera (1979). Más recientemente, los estudios agrupados bajo el nombre de «teoría de la frontera» cobran especial interés. $\mathrm{Al}$ respecto podrá consultarse: Michaelsen \& Johnson (2003).

7 Ferguson (1959) define el término diglosia e indica que este refiere a una situación lingüística estable en la que coexisten los dialectos primarios de una lengua y una variedad impuesta que difiere de la anterior en su grado de complejidad y codificación. Las funciones sociales de estas variedades están bien delimitadas. La primera se utiliza en las situaciones familiares, mientras que la segunda es la len- 
contacto, que generalmente son acompañadas de gran tensión social.

2. APLICACIÓN Y LÍMITES DE LOS MODELOS SOCIOLINGÜÍSTICOS (MYERS-SCOTTON, I993A Y I993B). LAS HETEROGENEIDADES ENUNCIATIVAS DE AUTHIERREVUZ (1984, 1995)

\section{I. El modelo de la lengua matriz y el modelo de la marcación (Myers-Scotton, 1993a, 1993b)}

La lingüista Myers-Scotton (1993a, 1993b) ha propuesto dos modelos que atienden a las motivaciones sociales y psicológicas y a las restricciones estructurales presentes en la alternancia de lenguas en comunidades multilingües. Su investigación, según es habitual en el ámbito de la sociolingüística, se circunscribe al habla exclusivamente.

$\mathrm{El}$ «modelo de la lengua matriz», que se edifica justamente a partir de la noción de lengua matriz, pone el acento en las restricciones morfosintácticas que operan sobre la alternancia de lenguas en el nivel intraoracional. Este modelo establece que los casos de alternancia de lenguas manifiestan el encuentro de una lengua matriz y de una o varias lenguas incrustadas. La lengua matriz provee el marco morfosintáctico del discurso en el que aparecen las emisiones con alternancia. Los postulados de este modelo indican que los sistemas de las lenguas en contacto no presentan el mismo nivel de actividad en una emisión determinada.

En forma complementaria, Myers-Scotton (I993b) propone el «modelo de la marcación» para abordar las funciones sociales de la alter-

gua de la «alta literatura», la educación formal y los asuntos administrativos. Sobre las derivaciones y reformulaciones de este concepto, podrá consultarse la obra de Ferguson (I959), Fasold (I996) y Kremnitz (1996). nancia de lenguas. El eje de este modelo es el concepto de marcación (markedness) que se refiere a la posibilidad que tienen los hablantes de producir emisiones que se ajusten más o menos a lo que se considera esperable en una situación comunicativa determinada. Así, algunas emisiones se perciben como «naturales» o «esperables», no marcadas; mientras que otras se alejan de las normas y acuerdos conversacionales y son, por tanto, marcadas. La noción de marcación se presenta como un continuo que permite clasificar las instancias que evidencian alternancia de lenguas como más o menos marcadas, en una situación social determinada en la que entra en juego la negociación de identidades en atención a la definición de los distintos conjuntos de derechos y obligaciones asociados a las lenguas intervinientes en la interacción.

En suma, a los efectos del análisis se destaca la importancia otorgada al concepto de marcación como continuo y a la percepción e interpretación del interlocutor en la asignación del valor de las formas que manifiestan alternancia de lenguas. Sin duda, este punto es de gran relevancia para evaluar el efecto que produce este recurso en el universo de un corpus literario, que incluye, por supuesto, la consideración del lector. Por otra parte, la noción de que el recurso a la alternancia de lenguas es evocador y constituye un sentido per se, independientemente de su valor referencial, por el simple hecho de ser empleado en una situación comunicativa, es determinante en la obra literaria. Por último, el constructo de la lengua matriz nos plantea un desafío particular: el análisis de casos que aquí proponemos indaga en las operaciones a través de las cuales es posible identificar la lengua matriz.

Ahora bien, aunque estas nociones constituyen sin duda un punto de partida ineludible en tanto explican cuestiones relativas al contacto 
de lenguas, entendemos que es necesario recurrir a un enfoque teórico que permita dar cuenta del empleo de la traducción literal que queda fuera de los modelos reseñados pero que se relaciona con las marcas que supone el empleo de dos lenguas simultáneamente. En este sentido y como se verá a continuación, sostendremos que la teoría de las heterogeneidades enunciativas de Authier-Revuz (1984, 1995) propone un marco teórico adecuado para abordar el análisis de este fenómeno lingüístico-cultural y acoger los aportes de los modelos sociolingüísticos mencionados.

\subsection{La teoría de las heterogeneidades enuncia- tivas de Jacqueline Authier-Revuz (1984, 1995)}

La teoría de las heterogeneidades enunciativas se ocupa de la constitución del discurso y de los procedimientos de representación por medio de los cuales esta constitución se manifiesta, atendiendo a los distintos niveles de la lengua: léxico-semántico, pragmático, morfosintáctico, gráfico y fonológico. En «Hétérogénéité(s) énonciative(s)», Authier-Revuz (I984) señala la necesidad de recurrir a enfoques que, situados en el exterior de la lingüística, han cuestionado la noción del sujeto como fuente y amo de su decir. La lingüista establece que su planteo de la heterogeneidad del sujeto y de su discurso se nutre de la noción bajtiniana de discurso como producto del interdiscurso y de la concepción del sujeto y su relación con su discurso, que toma de los trabajos de Freud y de Lacan.

Estos enfoques teóricos, que ponen en tela de juicio la concepción del sujeto como fuente autónoma de su decir, señalan que «toda palabra está determinada fuera de la voluntad de un sujeto, y que este, «más que hablar, es hablado»» (op. cit.: 99; traducción nuestra). La condición constitutiva de existencia del sujeto y de su discurso es, en efecto, ese plano exterior al cual tanto el sujeto como su discurso recurren para constituirse. En el caso de la literatura, es preciso recordar que esta afirmación encuentra cierto límite en la voluntad de un sujeto que se propone la tarea de la escritura creativa.

Retomando la teoría bajtiniana, AuthierRevuz (1995) señala la dimensión central de lo heterogéneo en la constitución de todo discurso. El dialogismo de Bajtín implica una «concepción dinámica de la producción del discurso bic et nunc, de su hilo y de su sentido, a partir de otros discursos» (op.cit:: 249; traducción nuestra). Al hablar o al escribir, utilizamos palabras que no nos pertenecen, que han vivido en el interior de otros discursos históricamente.

En su desarrollo, Authier-Revuz distingue entre heterogeneidad constitutiva y heterogeneidad mostrada. La heterogeneidad constitutiva alude a la conjunción de procesos que relacionan el exterior e interior del discurso continuamente en la constitución de ese discurso y del sujeto. La heterogeneidad mostrada, por su parte, define los modos de negociación por medio de los cuales se representa la constitución de ese discurso.

La heterogeneidad mostrada inscribe al otro en el discurso. A través de las formas de la heterogeneidad mostrada se altera la unicidad aparente del hilo del discurso. La heterogeneidad mostrada puede ser marcada o no marcada según existan formas que señalen aquello que es extraño al discurso o no. En el caso de la heterogeneidad marcada, el fragmento puede aparecer integrado o no al hilo del discurso, pero siempre existe una marca de su presencia en el discurso. Ejemplos de este tipo de heterogeneidad son: el discurso directo, el discurso indirecto, las palabras y las frases entrecomilladas, las glosas, etc. En el caso de la heterogeneidad mostrada no marcada, la alteridad, el fragmento designado como otro se presenta de manera más sutil, sin marca aparente. Algunas de las formas de la 
heterogeneidad mostrada no marcada son: el discurso indirecto libre, la ironía, los juegos de palabras, las metáforas y el pastiche.

70 Authier-Revuz indica que el interés en el estudio de las formas marcadas de la heterogeneidad mostrada reside en que estas especifican los parámetros y puntos de vista a partir de los cuales un discurso plantea explícitamente una alteridad con respecto a sí mismo. El uso de otra lengua, de otro registro discursivo, de otro discurso, la inclusión de otra palabra, de otro interlocutor - entre otras marcas - constituyen puntos de heterogeneidad.

Tal como ha señalado Bajtín (I982), la elección de una palabra tiene un poder evocador que nos remite a los contextos de enunciación en los que esta palabra ha sido usada anteriormente. En el caso de la narrativa de Cisneros, el empleo de ciertas expresiones en inglés, traducidas literalmente del español, y el uso de una sintaxis particular producen un nuevo juego de efectos y sentidos. Los contextos culturales e históricos que se ponen de relieve en esta narrativa evocan la historia de la comunidad chicana, su pasado mexicano, y su presente, en el que se conforma una nueva situación cultural, social y lingüística.

Como todo discurso, el discurso de Cisneros es constitutivamente heterogéneo. Sin embargo, es importante destacar que este discurso es doblemente heterogéneo ya que remite simultáneamente a dos interdiscursos culturales diferentes creando así un nuevo espacio enunciativo. Este artículo indaga en la construcción de la narrativa de Cisneros, específicamente en la caracterización del exterior al que recurre su discurso en el proceso de desterritorialización a través de las marcas de la traducción literal. La designación de un exterior específico es, a través de cada marca de distancia, una operación identitaria para el discurso. Por tanto, la zona de contacto entre estos planos, exterior e interior, es reveladora de la constitución de ese discurso.

$* * *$

Como ya hemos señalado en la Introducción, la operación de desterritorialización en la obra de Cisneros se materializa mediante distintas estrategias. Por un lado, el hecho de que las escrituras de minorías sean escritas en una lengua mayoritaria implica una operación de desterritorialización en sí. La literatura chicana en general expresa sentidos y contenidos que no pertenecen a la cosmovisión estadounidense típica. En segundo término y como se ha observado largamente, el contacto entre el español y el inglés, y específicamente el recurso a la alternancia de lenguas, se ha vuelto un rasgo característico de la narrativa chicana.

Las estrategias mencionadas han recibido gran atención por parte de lingüistas, críticos literarios y traductores; sin embargo, existe otro modo en el que la lengua se desterritorializa en el seno de la narrativa de Cisneros. Esta tercera estrategia aparece, como hemos dicho, esbozada en Rudin (1996). En Tender Accents of Sound. Spanish in the Chicano Novel in English, Rudin (1996) establece que los escritores chicanos, si bien no de manera habitual, construyen estrategias oblicuas para recrear el ámbito hispanohablante en sus textos. El autor distingue dos modalidades principales: el empleo de un inglés «hispanizado» («Hispanized English») y el empleo de un español ficticio. La primera estrategia refiere a los contextos de uso en los que el inglés ocasionalmente adopta la estructura del español o reproduce expresiones idiomáticas del español. La segunda estrategia alude a los casos en los que el narrador del relato o un personaje indican que los personajes hablan en español aunque sus palabras aparezcan en inglés. E1 investigador provee unos pocos ejemplos de 
estas estrategias pero no los sistematiza ya que no forman parte del interés principal que motiva su estudio.

Precisamente, este artículo busca examinar lo que Rudin denominó «Hispanized English». Para ello abordaremos el estudio de un conjunto de frases y estructuras que aparecen en inglés pero que manifiestan un fuerte contacto con el español. Según hemos afirmado, en la narrativa de Cisneros la desterritorialización de la lengua también se actualiza por medio de la traducción literal del español al inglés. En función de la hipótesis enunciada, el análisis del corpus intentará mostrar que estas instancias de contacto de lenguas particular constituyen formas y marcas de la desterritorialización que deben considerarse como casos especiales de alternancia de lenguas.

Dado, pues, que el objetivo principal de este artículo es indagar en la traducción literal en tanto estrategia de desterritorialización, que aparece en estrecha relación con la alternancia de lenguas en la escritura de Cisneros, y considerando que los modelos sociolingüísticos no incluyen el caso de la traducción literal dentro de sus descripciones, resulta necesario poner en relación nociones teóricas que, provenientes de distintos campos disciplinares, puedan dar cuenta de la complejidad enunciativa del corpus que nos proponemos analizar. Así, entendemos que en atención al vínculo que la traducción literal tiene con la alternancia de lenguas en el corpus, resulta operativo articular los aportes de los modelos sociolingüísticos reseñados (Myers-Scotton, I993a y I993b) bajo el marco general de la teoría de las heterogeneidades enunciativas de Authier-Revuz (1984, I995). En particular, se observará que la noción de marcación como continuo es necesaria para determinar los distintos grados de heterogeneidad manifiestos en la operación de desterritorializa- ción que se materializa a través de la traducción literal en el discurso de Cisneros. Según esta perspectiva, las marcas de la traducción literal presentes en el corpus pueden abordarse como 71 formas de la heterogeneidad mostrada marcada, que evocan un exterior al que el discurso recurre en el proceso de su constitución. Asimismo, como se apreciará, será crucial tener presente el constructo de la lengua matriz en el análisis del nivel morfosintáctico ${ }^{8}$.

\section{ALGUNAS FORMAS Y MARCAS DE LA TRA- DUCCIÓN LITERAL EN WOMAN HOLLE- RING CREEK (WHC)}

\section{I. Consideraciones generales}

Como hemos señalado, el corpus de este trabajo está constituido por frases y estructuras que registran cierta fijación y pertenece a la obra $W H C$ de la autora chicana Sandra Cisneros. WHC es una colección de relatos que da voz a personajes chicanos y mexicanos que habitan a ambos lados de la frontera geográfica, cultural y simbólica que se erige entre México y Estados Unidos. Esta frontera acompaña la experimentación literaria que se propone la autora en el trabajo de la escritura. Algunos de los cuentos semejan poemas y canciones populares mientras que otros recogen episodios trascendentales de la historia de México.

El discurso de Cisneros se mueve al menos entre dos universos lingüístico-culturales diferentes y plasma su escritura en la frontera en la que estos espacios pueden converger. Precisamente, la traducción literal se vislumbra como la marca sutil de un exterior al que recurre la prosa de la autora chicana en el proceso de su

\footnotetext{
8 Por otro lado, este enfoque teórico aporta las herramientas precisas para abordar las particularidades del nivel gráfico, que la autora chicana explota de manera singular.
} 
constitución. Al decir de Valenzuela (I996), la traductora de $W H C$ al español, esta particularidad de la escritura de Cisneros «le agrega sazón al caldo». Sin dudas, el empleo de estas frases idiomáticas traducidas literalmente del español obliga al inglés a expresar nuevos sentidos, sentidos que le resultan ajenos, contribuyendo así a la operación de desterritorialización de esa lengua en el seno de esta narrativa.

Asimismo, es relevante llamar la atención sobre una peculiaridad de la estrategia discursiva que examinaremos. El hecho de traducir literalmente formas que registran cierto grado de fijación contribuye a la conformación de un marco de discurso que es doblemente heterogéneo. Por un lado, el uso de frases fijas es un punto de heterogeneidad en sí. El recurso a estas frases estereotipadas evoca la voz colectiva cristalizada en la lengua. Pero en estos casos, a ello debe sumarse el hecho de que la voz colectiva, asociada al mundo latino, es evocada por medio de otra lengua, el inglés. De alguna manera, la autora chicana traduce elementos que dado su componente idiomático no pueden ser traducidos literalmente, desafiando así, tal vez, algunos de los principios rectores de la traducción, ya enunciados, entre otros, por Nida (1969) a propósito de la traducción literal de expresiones idiomáticas de la Biblia.

En atención a la hipótesis que sustenta este trabajo, es posible delimitar tres niveles de análisis principales: el nivel léxico-semántico, el nivel pragmático y el nivel morfosintáctico. Por su parte, los elementos del nivel gráfico acompañan los procesos que afectan a los otros tres niveles. En este artículo nos dedicaremos solo al estudio de algunos elementos de los niveles léxico-semántico y pragmático. De cualquier manera, a modo de ejemplo, en el nivel morfosintáctico, se distinguen usos marcados del caso genitivo, el uso del adjetivo posesivo y del artí- culo definido. Ejemplos como «the pink socks of the baby Amber Sue» $(W H C, 4)$; «she didn't run away as she imagined she might when she saw such things in the telenovelas» $(W H C, 47) \mathrm{y}$ «I'm sure my Abuelita has her own story, but it's not my place to ask» $(W H C, 28)$ ilustran algunas de las formas marcadas de la heterogeneidad vinculadas al nivel morfosintáctico. Como ya hemos indicado, el empleo de una sintaxis marcada por el recurso a la traducción literal señala un punto de heterogeneidad doble para el discurso. En el seno de la escritura de Cisneros la determinación del nombre opera generalmente desde el ámbito del español. Así, el uso marcado del caso genitivo, del artículo definido y del adjetivo posesivo, a través de la traducción literal, se vuelve una estrategia importante de desterritorialización en su escritura.

\subsection{El nivel léxico-semántico}

En todos los ejemplos que siguen hemos subrayado la expresión o estructura que será objeto de análisis. Toda cita de la obra se realizará siguiendo las características gráficas del original. En cada ejemplo, se indica entre paréntesis el nombre del cuento y la página que corresponde a cada caso:

I. «Salvador Late or Early.» («Salvador Late or Early», Io).

2. "And at the next full moon, I gave light." («Eyes of Zapata», 93).

3. «In the day, I could support the grief (...).» («Eyes of Zapata», 97).

4. "Yeah, sure, he was her sometime sweetheart, but what's that to a woman who's twenty and got the world by the eggs.» («La Fabulosa: A Texas Operetta», 62).

La frase del ejemplo I, «late or early», evoca, por medio de su traducción literal, la frase idiomática del español, «tarde o temprano». La 
frase aparece aquí en inglés pero con el orden propio del español9. Recordemos que existe en inglés un equivalente funcional para esa frase, sooner or later, que señala un grado comparativo ausente en la expresión del español. Por un lado, la frase traducida alude al español y a la cosmovisión relacionada con esa lengua, en la cual el primer elemento de la disyunción es «tarde» y no «temprano» como en inglés. Por otra parte, en relación con nuestra hipótesis central, el ejemplo resulta un caso sutil de alternancia de lenguas que afecta los niveles léxico y morfosintáctico. En este caso, el marco morfosintáctico no es el esperado - es marcado- porque pertenece a la lengua española y las unidades léxicas se reconocen como extrañas porque han sido traducidas literalmente. Con palabras pertenecientes a la lengua inglesa, Cisneros expresa de manera creativa y subversiva un nuevo sentido, que vincula el inglés con la cosmovisión latina, desterritorializándolo.

En el ejemplo 2, "gave light", la autora ha vertido palabra por palabra la expresión idiomática del español «dar a luz». En inglés, esta expresión tiene un equivalente funcional y dinámico, to give birth, que Cisneros decide no usar en su relato. Toda la oración, no solo la expresión idiomática, evoca la creencia popular perteneciente a la cosmovisión latina de que las mujeres embarazadas dan a luz en relación con los ciclos de la luna, «cuando cambia la luna». Esta creencia, al igual que tantas otras aludidas en la colección, no constituye un saber compartido entre el mundo latino y el mundo anglosajón. En otras palabras, el marco del discurso se asocia al mundo latino por medio de la traducción literal de la expresión idiomática «dar a luz». Resulta evidente que la

9 Alfonso y Eliggi (200r) mencionan este ejemplo, pero su análisis no excede la cuestión formal que atañe al orden morfosintáctico. marcación de lo heterogéneo se presenta como un continuo en el que es posible distinguir grados. Esta frase es muy marcada, no esperada, y requiere de la consideración del contexto para su interpretación correcta. En el interior de esta narrativa escrita en inglés, muchas veces, como muestran estos ejemplos, el inglés cumple funciones expresivas marcadas. Sin embargo, aun si la forma es marcada, es importante advertir que no lleva ninguna característica gráfica que la señale como extraña al lector monolingüe (anglohablante). Por lo general, la autora no incurre en agramaticalidades sintácticas pero parte de una lengua y llega a otra. Se evidencia así la desterritorialización de la lengua inglesa en el uso particular de la escritora chicana, que sutilmente conduce el inglés al terreno del español. Tanto en I como en 2 opera un cambio en el plano léxico-semántico, que sin duda corresponde al español. Así, la comprensión de estas frases (y de este discurso) depende en gran medida de la posibilidad del lector de interpretar que, en realidad, estas expresiones conducen hacia otro ámbito lingüístico-cultural, en el que hallan su origen y sentido. Se trata, pues, de emisiones que constituyen casos de alternancia de lenguas especiales.

En el ejemplo 3, "support the grief», al igual que en I y 2 , se evidencia un cambio en el plano idiomático. En este caso, a diferencia de los ejemplos anteriores, la autora no traduce la expresión del español literalmente, sino que produce una traducción homofónica; es decir, utiliza una palabra que es similar por sus características morfológicas y fonológicas a la forma española pero que no significa lo mismo, al estilo de un falso cognado. Esta frase, desprovista de sentido en inglés, evoca la idea de «soportar el dolor». El inglés registra distintas colocaciones que resultan equivalentes; por ejemplo, copel deal with the grief. Sin embargo, la autora ha 
entendido que estas formas no expresan el dolor que ella evoca, el dolor del pueblo mexicano que sufre por la guerra.

74 Por su parte, el ejemplo 4, «what's that to a woman who's twenty and got the world by the eggs», pone en escena un solapamiento de imágenes discursivas. Este ejemplo aparece citado en Brady (1999), quien, con gran claridad, argumenta que en $W H C$ el inglés y el español se manifiestan en una relación de contrapunto; es decir, se trata de voces que se combinan armoniosamente pero que, al mismo tiempo, se contraponen. En palabras de Arteaga (1997), estas voces compiten por el sentido. Su análisis del ejemplo señala la presencia de la traducción literal de la palabra «huevos» del español, que aparece en el texto como «eggs», en lugar de su equivalente funcional «balls». Y agrega que esta traducción produce una especie de broma satírica que alude simultáneamente a la complejidad de la traducción como a los diversos sentidos que se disparan cuando se dispone de dos lenguas. En esta relación de contrapunto descripta por Brady, la traducción literal tiene una parte crucial que la investigadora no explora en profundidad y que, consideramos, merece atención. Desde la perspectiva de análisis propuesta en este trabajo, que aborda los distintos exteriores que constituyen el discurso, se puede indagar en otros aspectos centrales a la conformación de esta frase.

Así, con respecto a la cuestión de la traducción, es preciso establecer, en primer lugar, que se trata de la traducción literal de toda una expresión idiomática y no solo de uno de sus términos. En efecto, la escritora está aludiendo aquí a la frase, «tener agarrado el mundo por las bolas", no registrada generalmente en los diccionarios de la lengua española, que vierte en su prosa a partir de una traducción palabra por palabra, evocando así un nuevo sentido lingüístico-cultural, que se asocia a lo latino. Es decir, el término «eggs» aparece como una traducción literal de «huevos», que, conviene agregar, en la frase del español tiene el sentido de «testículos» y no de «huevo de gallina», como en la versión en inglés que provee la autora, imponiendo así una nueva orientación para la palabra «eggs» en su discurso. Ahora bien, en el contexto de uso en el que aparece la frase, se activan también otros sentidos que aluden al mismo origen cultural y que resuenan en el lector bilingüe y bicultural inmediatamente. Es posible pensar en la frase empleada como una derivación del refrán popular de sentido equivalente en español: «tener a Dios agarrado de/por las barbas»" En la alusión al español, que se vislumbra a través de la traducción literal, se activan estos otros sentidos que quedan a disposición del lector de manera simultánea. Y en la interacción de los planos idiomático y léxico-semántico se registra, al igual que en los ejemplos anteriores, una instancia de alternancia de lenguas.

De este modo y según hemos observado en los ejemplos, la traducción literal de estas frases se propone como un caso especial de alternancia de lenguas, si consideramos este fenómeno en sentido amplio. Los ejemplos dan cuenta de un cambio de planos o niveles, que señala un exterior constitutivo para este discurso fronterizo, que recurre permanentemente a los elementos lingüístico-culturales del español pero los expresa en inglés. Y debemos notar la naturaleza también fronteriza de ese exterior. En el proceso de su constitución el discurso Cisneros apela al acervo lingüístico-cultural del español que aparece traducido literalmente.

Por último, la introducción de estas frases idiomáticas constituye un punto de heteroge-

ro Cisneros hace un uso particular de refranes y proverbios que vierte en su prosa, traducidos literalmente del español. El empleo de paremias se destaca en Caramelo or Puro Cuento, su última novela. 
neidad fuerte ya que, a pesar de ser formas muy marcadas, no interrumpen la unicidad aparente del discurso. Desde el marco conceptual que ofrece la teoría de las heterogeneidades, estas frases son metáforas cristalizadas que, originadas en el plano del español y del mundo latino, han sido traducidas literalmente. Así, comparten rasgos con las formas marcadas y no marcadas de la heterogeneidad mostrada. De cualquier modo, es importante advertir que el procedimiento principal aquí es la traducción literal, que constituye indudablemente una forma de la heterogeneidad marcada.

\subsection{El nivel pragmático}

\subsection{Formas de vocativo}

Desde el punto de vista pragmático-discursivo, los vocativos son nombres o pronombres cuya función es apelar al oyente. Cuando se utilizan nombres comunes, estos pueden denotar la edad, el rango, la profesión o la relación que existe entre las personas que intervienen en una situación de comunicación dada. Asimismo, el uso del vocativo puede poner de manifiesto diversas actitudes: de afecto, de respeto, de solidaridad, de atención. En cuanto a sus propiedades sintácticas, es relevante mencionar que estas formas no concurren con artículos pero sí con adjetivos posesivos o interjecciones (AlonsoCortés, 1999). En ciertos casos, la construcción del vocativo puede combinar más de uno de los aspectos mencionados. Entre las clases de nombres que se emplean generalmente como vocativos están los que denotan trato íntimo. En relación con su estatuto dentro del enunciado, es preciso notar que las expresiones vocativas tienen cierta independencia sintáctica y constituyen un plano autónomo diferente del plano del enunciado (Quirk et al., 1985). Así, estas formas funcionan como islas sintácticas que se sitúan en el plano de la enunciación y no del enunciado.

En la mayoría de las lenguas, como señala Levinson (1983), existen formas vocativas que resultan muy idiosincrásicas y complejas ${ }^{\mathrm{II}}$. Es de interés recordar que, dada su naturaleza idiomática, las expresiones fijas generalmente no aceptan la prueba de la traducción palabra por palabra. Cada comunidad lingüístico-cultural crea sus propias formas lingüísticas en las que plasman una cosmovisión particular. Según se apreciará en los ejemplos, la autora chicana ha traducido literalmente las expresiones de vocativo del español al inglés, produciendo una marca particular que contribuye a la estrategia de desterritorialización que este articulo describe.

I. «Ay, my life, remember?» (WHC, 89).

2. "My sky, my life, my eyes. Let me look at you.» (WHC, $\mathrm{II} 3)$.

3. "You opened your arms, my heaven, but kept your precious heart locked.» (WHC, I36). 4. " My soul, did I hurt you?» (WHC, I54).

La mayoría de las expresiones vocativas de WHC se encuentran en tres cuentos: «Eyes of Zapata», «Tin Tan Tan» $\mathrm{y}$ «Bien Pretty». Estos cuentos se proponen como historias de amor en las que el diálogo juega un papel constitutivo primordial. En el primer caso, la narradora, Inés Alfaro, dialoga imaginariamente con su amante, el líder mexicano Emiliano Zapata. En los otros dos relatos, la trama gira en torno a la relación amorosa que establecen los personajes Rogelio Velasco y Lupe Arredondo.

Por otro lado, los ejemplos citados devienen formas muy marcadas cuyo efecto merece consideración. Su empleo evoca el eco de las

II Para ampliar este tema, podrá consultarse Levinson (1983), que aborda los distintos tipos de deixis. 
situaciones de enunciación en las que, traducidas literalmente, estas expresiones funcionan como vocativos. Es necesario advertir que solo el lector bilingüe y bicultural de estos textos, que tenga el español como marco de referencia, podrá discernir la función de estas formas. El lector monolingüe (anglohablante), en cambio, no podrá distinguir en todos los casos que estas expresiones cumplen la función de vocativos. Muy hábilmente, la autora obliga al lector a cuestionar su lectura y su propia lengua, que aparece como extraña al entrar en contacto con otro sistema lingüístico-cultural.

El contacto lingüístico-cultural que se da entre los personajes mencionados se materializa, entre otras marcas, en estas formas fronterizas, que se proponen como zonas lingüístico-culturales ideales para explorar las fisuras entre los sistemas. El caso del vocativo pone en juego la necesidad de nombrar al otro en el propio discurso. Los ejemplos muestran que la marca de la heterogeneidad reside tanto en las elecciones del nivel léxico-semántico como en el componente pragmático de la lengua. La evocación del ser amado se experimenta desde la cosmovisión latina, que opera como marco de discurso, pero se expresa en un inglés que, al llevar la marca de la traducción literal, aparece como extraño. Así, el inglés «pierde territorio» al expresar nuevos sentidos. En esta narrativa, el mundo del afecto, el plano de lo más íntimo encuentra el origen de su expresión en un universo que siempre se asocia al español.

Asimismo, el empleo de estas formas de vocativo también da cuenta del carácter doblemente heterogéneo del discurso de Cisneros. Por un lado, el uso de vocativos alude a los elementos cristalizados de la lengua y por el otro, la traducción literal de estos elementos evoca el español. El discurso reenvía a un exterior que

resulta esencial para su constitución. Ese exterior, marcado por la traducción literal, da cuenta de que bajo la apariencia del inglés en estas formas vibra el español. En los ejemplos citados el cambio fundamental opera en el nivel pragmático instituyendo así instancias especiales de alternancia de lenguas.

\subsubsection{Coletillas interrogativas (question tags)}

Por lo general, en inglés, las coletillas interrogativas son frases que se agregan al final del enunciado y su estructura gramatical está determinada por la estructura de la oración principal, a la cual se refieren. Dada su función, las coletillas interrogativas pertenecen al plano de la enunciación, aun si su estructura se define a partir de las características del enunciado. En la mayoría de los casos, la formación de la coletilla interrogativa coincide con el tiempo, modo y aspecto verbal del verbo de la oración principal. Con frecuencia, la coletilla interrogativa invierte la polaridad de la oración principal. Es negativa, si la oración principal es positiva y viceversa. Dada su estructura de diálogo, el cuento «Eyes of Zapata» abunda en este tipo de marcadores: «You knew before any of us, didn't you?»; «You're stubborn as he was, aren't you?»; «You don't like me to talk about my father, do you?».

Por su parte, las coletillas del español comparten la mayoría de las características enunciadas, excepto por su estructura, que siempre es la de un adverbio o interjección en español. Según el dialecto del español de que se trate, es frecuente encontrar las siguientes coletillas: «jah?», «¿sí?», «ंno?», «ंeh?», «نverdad?», etc.

Resulta importante advertir que estas formas se consideran marcadores discursivos, en tanto la pregunta que entrañan va dirigida a algún 
aspecto del estado de la conversación ${ }^{\mathrm{I} 2}$, más que a la búsqueda de información en sí. En otras palabras, la coletilla interrogativa no interroga acerca del contenido proposicional del enunciado sino acerca de la relación que se establece entre los participantes de una conversación respecto de ese contenido. Generalmente, su uso indica la búsqueda de confirmación de un supuesto del hablante (Escandell-Vidal, 1999). En el caso del texto escrito, su inclusión crea la necesidad de relectura, como se verá más adelante.

I. "Always muy enamorado, no?» («Eyes of Zapata», 89).

2. "(...) you've always prided yourself in being independent, no?» ( "Eyes of Zapata», 95).

3. "You have your pastimes. That's how it's said, no?» («Eyes of Zapata», Ioo).

4. "All members of one army against us, no?" («Eyes of Zapata», IoI)

5. «You don't belong to anyone, no?» («Eyes of Zapata», IIo).

Los ejemplos pertenecen al cuento «Eyes of Zapata» ya mencionado. Es posible analizar las coletillas de los ejemplos como casos de heterogeneidad doble, si el adverbio «no» se interpreta como un elemento fijo del español que ha sido traducido literalmente. Sin embargo, en este caso no se trata necesariamente de una traducción literal; podría pensarse en el «no» de las coletillas como en una instancia de alternancia de lenguas. De cualquier manera, el estatuto del español no resulta absolutamente evidente. $\mathrm{Su}$ presencia podría haberse reforzado mediante la marca de la bastardilla y del signo de interroga-

I2 En el discurso oral, la entonación generalmente determina la función e interpretación de estas frases. Al respecto podrá consultarse Quirk et al, I985: 8I2. ción de apertura, como sucede en otros lugares de la colección ${ }^{13}$. Aquí, la coletilla se expresa mediante un adverbio que comparten las dos lenguas, por lo que su estatuto es aun más difícil de determinar. Es dable afirmar que este tipo de casos se erige justamente en la fisura, en la frontera que separa y une los dos sistemas lingüístico-culturales.

En el discurso escrito las coletillas obligan muchas veces a reconsiderar lo expuesto en los enunciados que las anteceden. Es preciso, entonces, reparar en el efecto que estas marcas producen en términos generales en el caso del relato «Eyes of Zapata». Al plantearse como un diálogo imaginario entre los amantes Inés Alfaro y Emiliano Zapata, el tejido del cuento recurre constantemente a marcas que aluden a su relación amorosa. El personaje de Zapata, que supuestamente duerme mientras Inés transita distintos momentos de su historia personal y de la historia de México, jamás responde las preguntas de Inés. A medida que el relato progresa, la recurrencia de estas marcas termina por colocar al lector en la situación de Zapata que ahora «debe responder» de algún modo a todos los interrogantes que Inés plante ${ }^{\mathrm{I}}{ }^{4}$. Es el lector quien cierra el sentido en última instancia.

Asimismo y en relación con el punto anterior, debe observarse que el uso de las coletillas exige que el lector vuelva a plantearse el contenido proposicional del texto a cada instante. Dado el carácter marcadamente fronterizo de estas coletillas, la relectura se efectúa desde un marco de lectura que aporta la pragmática del español,

13 A modo de ejemplo, véase en el cuento «Bien Pretty» la aparición de coletillas interrogativas en español con la puntuación correspondiente a esa lengua ( $D$ De poeta y de loco todos tenemos un poco, ¿̇no?»).

I4 La función de estas coletillas se puede equiparar a la figura del narrador intrusivo que se dirige al lector por medio del pronombre de segunda persona. 
lengua en la que estas formas son variantes esperadas, no marcadas. Se observa, además, que las estrategias discursivas se construyen en 78 coincidencia con un punto de vista político: la razón de ser del relato es probablemente contar un episodio histórico trascendental para el pueblo mexicano desde una nueva perspectiva.

En el primero de los ejemplos, «Always muy enamorado, no?», el marco morfosintáctico corresponde al español en su totalidad y se percibe, por tanto, como una traducción literal. Desde el comienzo de la oración, opera un cambio que afecta al nivel morfosintáctico, antes de que aparezca el español en todo su esplendor, reforzado con la marca de la letra bastardilla. E1 caso específico de la coletilla es interesante porque continúa la expresión del español evocado en el enunciado que la precede. Si bien la discursividad se construye desde lo latino en este caso, la puntuación de la coletilla es la esperada en inglés, señalando una vez más el carácter fronterizo de este discurso.

En el segundo ejemplo, «(...) you've always prided yourself in being independent, no?», el primer elemento no esperado es la preposición in que rige generalmente el sustantivo pride pero no así el verbo to pride. El régimen del verbo se construye habitualmente con las preposiciones on y upon. A diferencia del caso anterior, la lengua matriz en la primera parte de este ejemplo es el inglés. Sin embargo, la adición de la pregunta al final, en la que aparece el marcador discursivo del español, obliga a reconsiderar el todo desde la cosmovisión que se asocia a esa lengua, y no al inglés.

E1 tercer caso, "You have your pastimes. That's how it's said, no?», también presenta particularidades. En primer lugar, la marca de la bastardilla en "pastimes» plantea una alteridad implícita, que se hace explícita con el comentario metadiscursivo que sigue en el

enunciado siguiente. Es relevante comentar que en inglés esta palabra no se asocia con el sentido de affaire o amorío. E1 exterior que señala la bastardilla evoca, entonces, el español, en el que la palabra "pasatiempo" puede interpretarse de esa manera. Esta evocación se ve reforzada por el comentario que señala un lugar de enunciación en el que "pastimes» funciona como «amorío». Se trata, una vez más, de una instancia en la que ha cambiado el componente idiomático y el inglés debe interpretarse desde el español. El recurso a otra lengua mediante la traducción literal impone en este caso la alusión a un uso colectivo que es esperado, no marcado, en los contextos en que esa lengua se utiliza. Por medio de su empleo, se activa, como señala Myers-Scotton, el conjunto de valores, derechos y obligaciones que se asocian con el uso de esa lengua. La coletilla interrogativa al final de la oración instaura otro exterior, que es similar al de los casos anteriores.

Resulta también de interés advertir una característica común a los tres ejemplos. La aparición de la forma marcada está de una u otra manera preanunciada por otra marca: la presencia del español en el primer ejemplo, el uso marcado de una preposición en el segundo y el recurso al componente idiomático de otra lengua en el tercer caso.

Por su parte, los ejemplos cuatro, «All members of one army against us, no?», y cinco, «You don't belong to anyone, no? ?, son en gran medida similares al segundo ejemplo. El carácter dinámico de la lengua matriz se hace evidente en ambos ejemplos. La oración se construye desde un marco morfosintáctico no marcado para el inglés pero sobre el final se produce un cambio, introducido por la coletilla, que obliga al lector a evaluar lo dicho desde una nueva perspectiva, la del exterior que se asocia al mundo hispano. 


\section{CONCLUSIONES}

Este artículo continúa una línea de investigación esbozada en Rudin (1996) respecto de ciertos usos especiales del inglés en el seno de la narrativa chicana. En efecto, a lo largo de estas páginas hemos examinado el empleo del «inglés hispanizado» en la colección de relatos WHC de Cisneros. Desde el marco que aporta la teoría de las heterogeneidades enunciativas de Authier-Revuz, hemos evaluado la relación que la traducción literal guarda con la operación de desterritorialización y la alternancia de lenguas. En el interior de esta narrativa la traducción literal del español al inglés juega un papel primordial. Por un lado, resulta un medio para la desterritorialización de la lengua y, por el otro, constituye un caso especial de alternancia de lenguas, en tanto su presencia es una marca que deja entrever alguno de los niveles de la otra lengua.

En cuanto al estudio de casos, el análisis se ha centrado en dos niveles: el léxico-semántico, en el que hemos señalado el empleo particular de un conjunto de frases idiomáticas, y el pragmático, en el que abordamos el examen de expresiones de vocativo y de las coletillas interrogativas. Con respecto al nivel léxico-semántico, las formas cristalizadas generalmente aparecen vinculadas a temas y cuestiones culturales de las comunidades chicana y mexicana. La traducción literal de estas formas produce un efecto de extrañeza en el lector y se constituye, a medida que la obra progresa, en un vehículo que permite el ingreso a la otra cultura, aquella que vive bajo la apariencia del inglés. En el nivel pragmático, el hecho de que los elementos estudiados aludan a la relación interpersonal entre los personajes de los relatos resulta una elección muy significativa. En todos los casos, se trata de elementos que, enmarcados en la enuncia- ción, instauran zonas heterogéneas en el relato creando así espacios discursivos que señalan la frontera cultural y lingüística que $W H C$ explora de manera singular.

Asimismo, el análisis de casos ha mostrado que los cambios de nivel examinados constituyen formas marcadas de la heterogeneidad y casos especiales de alternancia de lenguas. Esta característica, que recorre todos los casos del corpus, permite afirmar que en estos ejemplos se materializa la alternancia de lenguas de una forma particular, no contemplada hasta ahora en los modelos sociolingüísticos. Así, queda de manifiesto la necesidad de ampliar el concepto de alternancia de lenguas para que pueda incorporarse la consideración de instancias de traducción literal, que denotan el contacto de dos sistemas lingüístico-culturales.

Las estrategias discursivas estudiadas juegan un papel central en el proceso de desterritorialización de la lengua en la narrativa de Cisneros. Y en los casos analizados, el hilo conductor de estos procedimientos es la traducción literal. En efecto, en el entramado discursivo de estos relatos la traducción literal se presenta como un procedimiento constitutivo que genera efectos particulares en el lector. Por último, el análisis permite advertir que la traducción literal de ciertas expresiones y estructuras pone a prueba la traducibilidad, en tanto muestra que los discursos evocados por estas instancias creadas por la autora no podrían evocarse mediante palabras asociadas al acervo lingüístico-cultural del inglés. Más aún, las escenas enunciativas que ponen en marcha los procesos de traducción en esta obra pueden considerarse como un fenómeno de contacto de lenguas. La traducción como proceso puede definirse como un camino entre dos culturas. En tanto producto, un texto traducido deja entrever, de manera sutil, el camino recorrido. 
Este punto merecerá nuestra atención en trabajos posteriores.

Retomando los términos de la Introducción 80 respecto de la posición de Cisneros como escritora, es importante advertir que la traducción se constituye, como bien apunta de Zavalía (2000), en una estrategia de supervivencia fundamental. Según Cisneros misma ha declarado en diversas entrevistas (Oliver-Rotger, 2000), su misión como escritora es contribuir a acortar distancias $\mathrm{y}$ traducir, tarea que le resulta fascinante. Ahora bien, en $W H C$, más que en ninguna otra obra, Cisneros «se traduce», en el sentido poscolonial del término, pero le exige al lector que haga su parte y que, a su turno, también «se traduzca» para poder completar el nuevo sentido cultural que su narrativa quiere expresar. Acaso se trate de una búsqueda intensa de lo que Ricoeur (2005) denominó hospitalidad lingüistica, cuyo régimen es el de una correspondencia sin adecuación. Por ello, en tanto escritora que traduce, la postura que adopta Cisneros se asocia a la extranjerización en $W H C$. Prontamente, debemos advertir que es posible trazar una evolución en este posicionamiento a lo largo de su obra narrativa, tema que será objeto de investigaciones futuras.

RECIBIDO EN JULIO DE 2008 ACEPTADO EN NOVIEMBRE DE 2009 VERSIÓN FINAL DE JUNIO DE 2009

\section{REFERENCIAS BIBLIOGRÁFICAS}

Alfonso, N.L. y M. G. Eliggi (200I). «Lengua y poder en algunos cuentos de Sandra Cisneros». En Estudios literarios sobre la cultura chicana. Publicación del Depto. de Lenguas Extranjeras, Facultad de Ciencias Humanas, Universidad Nacional de La Pampa, pp.8I-87.

Alonso-Cortés, A. (1999). "Las construcciones exclamativas. La interjección y las expresiones vocativas». En I. Bosque \& V. Demonte (dirs.). Gramática descriptiva de la lengua española. Madrid: Espasa Calpe, pp. 3993-4050.

Arteaga, A. (1997). «An Other Tongue». En A. Arteaga. Chicano Poetics. Heterotexts and Hybridities. Australia, EEUU y Reino Unido, Cambridge University Press, pp. 68-9o.

Ashcroft B. et al. (2002 [1989]). The Empire Writes Back. Cornwall: Routledge, $2^{\circ}$ edición.

Authier- Revuz, J. (1984). "Hétérogénéité(s) énonciative(s)». Langages, 73, pp. 98-III.

- (1995). Ces mots qui ne vont pas de soi. Boucles réflexies et non-coïncidences du dire. París: Larousse.

Barrera, M. (1979). Race and Class in the Southwest. Notre Dame IN: University of Notre Dame Press.

Bajtín, M. (1982 [1979]). Estética de la creación verbal. México: Siglo XXI, trad. de T. Bubnova.

Bhabha, H. (2004 [1994]). The Location of Culture. New York: Routledge Classics.

Brady, M.P. (1999). «The Contrapuntal Geographies of Woman Hollering Creek and Other Stories». American Literature, 7I, pp.II7-I50.

Cantero M. y P. Stewart (2003). «La creación del español mestizo en la literatura chicana: identidad y elección lingüística». En M. P. Gutiérrez \& J. Coloma Maestre (eds.). El español, lengua del mestizaje y la interculturalidad. Madrid: CDRom.

Cintron, Z. A. y E. Mendieta- Lombardo (1995). «Marked and Unmarked Choices of Codeswitching in Bilingual Poetry». Hispania, 78, pp. $565-572$.

Cisneros, S. (1991). Woman Hollering Creek and Other Stories. Nueva York: Vintage Contemporaries.

- (1996). El arroyo de la Llorona y otros cuentos. Nueva York: Vintage Español.

- (2002). Caramelo or Puro Cuento. Nueva York: Vintage Contemporaries.

Deleuze, G. y F. Guattari (1975). Kafka. Por una literatura menor. México: Era, trad. de J. Aguilar Mora.

Escandell- Vidal, M. A. (I999). «Los enunciados interrogativos. Aspectos semánticos y pragmáticos». En I. Bosque \& V. Demonte (dirs.). Gramática descriptiva de la lengua española. Madrid: Espasa Calpe, pp. 3929-3991. 
Fasold, R. (1996). La sociolingüistica de la sociedad. Introducción a la sociolingüistica. Madrid: Visor, trad. de M. España Villasarte y J. Mejía Alberdi.

Ferguson, C. A. (1959). «Diglossia». Word, 15, pp. 325-340.

García Vizcaíno, M.J. (2008). «Cisneros' CodeMixed Narrative and its Implications for Translation». Mutatis Mutandis, vol. I, 2, pp. 212- 224.

Irizarry, G. (200I). "Travelling Textualities and Phantasmagoric Originals: A Reading of Translation in Three Recent SpanishCaribbean Narratives». Ciberletras: Revista de critica literaria $y$ de cultura, 4 . <http:// www.lehman.cuny.edu/ciberletras/vo / Irizarry.html>. [Consulta: 20 de mayo de 2006].

Jiménez Carrá, N. (2005). «Estrategias de cambio de código y su traducción en la novela de Sandra Cisneros Caramelo or Puro Cuento». TRANS, 9, pp. 37-59.

Kremnitz, G. (1996). «Diglossie». En H. Goebl, P. Nelde, Z. Starý y W. Wöllck (eds.). Kontaklinguistik/Contact Linguistics/Linguistique de contact. Berlin/New York: Walter de Grutyer. vol. I, pp. $245^{-257}$.

Leal, L. y M. M. Martín-Rodríguez. (2006 [I996]). «La literatura chicana». En R. González Echevarría y E. Pupo-Walker (eds.). Historia de la literatura bispanoamericana. Tomo II. El siglo XX. Madrid: Gredos, pp. 557-585.

Levinson, S (1983). Pragmatics. Cambridge: Cambridge University Press.

Lipski, J. (I982). "Spanish-English Language Switching in Speech and Literature: Theories and Models». The Bilingual Review/La Revista Bilingüe, 9, pp.I9I-2I2.

Martín-Rodríguez, M.M. (I996). «The Global Border: Transnationalism and Cultural Hybridism in Alejandro Morales's The Rag Doll Plagues». The Bilingual Review/La revista bilingüe, 20 , pp. 86-98.

Michaelesen S. y E. D. Johnson (2003). Teoría de la frontera. Los limites de la politica cultural. Barcelona: Gedisa.

Muñoz Jr., C. (I997). «The Quest for Paradigm: The Development of Chicano Studies and Intellectuals». En A. Darder, R. Torres \& H. Gutiérrez (eds.). Latinos and Education. Nueva York: Routledge.
Myers-Scotton, C. (1993a). Duelling languages. Grammatical Structure in Codeswitching. Oxford: Clarendon Press.

- (1993b). Social Motivations for Codeswitching. Evidence from Africa. Oxford: Clarendon Press.

Newmark, P. (I988 [1982]) Approaches to Translation. Gran Bretaña: Prentice Hall International (UK) Ltd.

Nida, E. (1969). «Science of Translation». Language, Vol. 45, 3, pp. 483-498.

Oliver-Rotger, M. A. (2004 [2000]). «An Interview with Sandra Cisneros». Voices from the Gaps. Department of English, University of Minnesota, Minneapolis. <http://voices.cla.umn.edu/vg/ index.html > [Consulta: Io de octubre de 2006.]

Quirk, R. et al. (1985). A Comprehensive Grammar of the English Language. Londres: Longman.

Ricoeur, P. (2005). Sobre la traducción. Buenos Aires: Paidós, traducción y prólogo de Patricia Willson.

Rudin, E. (1996). Tender Accents of Sound. Spanish in the Chicano Novel in English. Tempe, Ariz.: Bilingual Press/Editorial Bilingüe.

Schleiermacher, F. (I994 [I8I3]) «Sobre los diferentes métodos de traducir». En M. A.Vega (ed.). Textos clásicos de teoría de la traducción. Madrid: Cátedra, pp. 224-235.

Soler-Espiauba, D. (200I). «Mestizaje lingüístico: funciones del español y del inglés en la expresión de los sentimientos en la narrativa hispana femenina de EE. UU.»: En B. de Jonge (ed.). Estudios de lingüística española, vol. I3. <http://elies.rediris. es/eliesiz/soler.htm> [Consulta: Io de octubre de 2006.]

Spoturno, M. L. (2008). «Fronteras y heterogeneidad. Un acercamiento al estudio del discurso fronterizo en Woman Hollering Creek de Sandra Cisneros». Páginas de Guarda. Revista de lenguaje, edición y cultura escrita, 6, pp.III-I29.

Staples, R (1976). «Race and Colonialism: The Domestic Case in Theory and Practice». The Black Scholar, 7, pp. 37-48.

Trivedi, H. (2005). «Translating Culture vs. Cultural Translation». www. grstmeridian.org. vol. 4, I, University of Iowa. <http://iwp.uiowa.edu/9Ist/ vol4_nI/index.html> [Consulta: 20 de noviembre de 2007.]

Tymoczko, M. (2000). «Translations of Themselves: The Contours of Postcolonial Fiction». En S. Simon y P. St. Pierre (eds.). Changing the Terms.
81 
Translating in the Postcolonial Era. Ottawa: University of Ottawa Press, pp.I47-I66.

Zavalía, J. de (2000). "The Impact of SpanishAmerican Literature in Translation on U.S. Latino Literature». En S. Simon y P. St. Pierre (eds.). Changing the Terms. Translating in the
Postcolonial Era. Ottawa: University of Ottawa Press, pp.187-206.

Valenzuela, L. (I996) «Ni chicha, ni limonada: Tras bambalinas con la traductora». Epílogo de S. Cisneros. El arroyo de la Llorona y otros cuentos. Estados Unidos: Vintage Español, pp. I87-rgr. 